\title{
Inhibition of receptor-interacting protein kinase 1 improves experimental non-alcoholic fatty liver disease
}

Amine Majdi, ${ }^{1,2}$ Lynda Aoudjehane, ${ }^{1,2 *}$ Vlad Ratziu, ${ }^{2,3,4}$ Tawhidul Islam,,${ }^{5,6}$ Marta B. Afonso, ${ }^{5}$ Filomena Conti, ${ }^{1,2,7}$ Taïeb Mestiri, ${ }^{1,2}$ Marie Lagouge, ${ }^{4}$ Fabienne Foufelle, ${ }^{4}$ Florine Ballenghien, ${ }^{1,2}$ Tatiana Ledent, ${ }^{1}$ Marthe Moldes ${ }^{1,2}$, Axelle Cadoret, ${ }^{1,2}$ Laura Fouassier, ${ }^{1,2}$ JeanLouis Delaunay, ${ }^{1,2}$ Tounsia Aït-Slimane, ${ }^{1,2}$ Gilles Courtois, ${ }^{8}$ Bruno Fève, ${ }^{1,2,9}$ Olivier Scatton, ${ }^{1,10}$ Carina Prip-Buus, ${ }^{6}$ Cecília M. P. Rodrigues, ${ }^{5}$ Chantal Housset, ${ }^{1,2,11}$ and Jérémie Gautheron ${ }^{1,2, \#}$

${ }^{1}$ Sorbonne Université, Inserm, Centre de Recherche Saint-Antoine (CRSA), Paris, France; ${ }^{2}$ Institute of Cardiometabolism and Nutrition (ICAN), Paris, France; ${ }^{3}$ Assistance PubliqueHôpitaux de Paris (AP-HP), Pitié-Salpêtrière Hospital, Department of Hepatology, Paris, France; ${ }^{4}$ Sorbonne Université, Inserm, Centre de Recherche des Cordeliers (CRC), Paris, France; ${ }^{5}$ Research Institute for Medicines (iMed.ULisboa), Faculty of Pharmacy, Universidade de Lisboa, Lisbon, Portugal; ${ }^{6}$ Université Paris Descartes, Institut Cochin, Inserm, CNRS, Paris France; ${ }^{7}$ AP-HP, Pitié-Salpêtrière Hospital, Department of Medical Liver Transplantation, Paris, France; ${ }^{8}$ Inserm, CEA, Institut de Biosciences et Biotechnologies (BIG), Grenoble, France; ${ }^{9} \mathrm{AP}-\mathrm{HP}$, Saint-Antoine Hospital, Department of Endocrinology, Paris, France; ${ }^{10} \mathrm{AP}-\mathrm{HP}$, Pitié-Salpêtrière Hospital, Department of Hepatobiliary Surgery and Liver Transplantation, Paris, France; ${ }^{11}$ AP-HP, Saint-Antoine Hospital, Department of Hepatology, Paris, France. *Authors sharing first authorship

\#To whom correspondence should be addressed: Dr. Jérémie Gautheron, Centre de Recherche Saint-Antoine (CRSA), Sorbonne Université, 27 rue Chaligny 75571 Paris Cedex 12, France. Email: jeremie.gautheron@inserm.fr

Key words: Necroptosis; RIPK1; MLKL; NAFLD; NASH; Steatosis.

Conflict of interest declaration: The authors have no conflict of interest to declare. 


\section{Lay summary}

Non-alcoholic fatty liver disease (NAFLD), the most frequent liver disease, currently lacks pharmacological treatment. Herein, we show that RIPK1, a gatekeeper of the necroptosis pathway, known to be activated in NAFLD, can be inhibited by RIPA-56 to reduce not only liver injury, inflammation and fibrosis but also steatosis in experimental models, via the control of mitochondrial respiration. In patients with NAFLD, inflammatory activity triggers RIPK1 release in the systemic circulation. These results highlight the potential of RIPK1 as a therapeutic target in NAFLD.

\section{Highlights}

- RIPA-56 reduces hepatic inflammation and fibrosis in dietary obese mice.

- RIPA-56 reverses steatosis and dampens body weight gain in obese mice.

- RIPK1 regulates triglyceride content in hepatocytes through activation of MLKL, which controls mitochondrial biomass and activity.

- RIPK1 and MLKL are significantly increased in the serum of patients with NASH.

- RIPK1/MLKL targeting represents a promising strategy for NASH treatment. 


\section{List of abbreviations}

ACOX1, acyl-coa oxidase 1; ALT, alanine aminotransferase; AML-12, alpha mouse liver-12; BrdU, bromodeoxyuridine; BSA, bovine serum albumin; CPT1A, carnitine palmitoyltransferase 1A; CS, citrate synthase; DAPI, 4',6-diamidino-2-phenylindole; DMEM, Dulbecco's modified Eagle's medium; DMSO, dimethylsulfoxide; FCCP, carbonyl cyanide4-(trifluoromethoxy) phenyl-hydrazone; FFA, free fatty acid; GAPDH, glyceraldehyde-3phosphate dehydrogenase; H\&E, haematoxylin and eosin; HFD, high-fat diet; HMBS, hydroxymethylbilane synthase; HPRT, hypoxanthine guanine phosphoribosyl-transferase; IDO, indoleamine 2,3-dioxygenase; MLKL, mixed lineage kinase domain-like; MRC, mitochondrial respiratory chain; MTT, 3-(4,5-dimethylthiazol-2-yl)-2,5-diphenyltetrazolium bromide; MTTP, microsomal triglyceride transfer protein; NAFLD, non-alcoholic fatty liver disease; NASH, non-alcoholic steatohepatitis; NCD, normal chow diet; OCR, oxygen consumption rate; PA, palmitic acid; PBS, phosphate buffered saline; PGC1A, peroxisome proliferator-activated receptor gamma coactivator-1 alpha; $\mathrm{PHH}$, primary human hepatocytes; OA, oleic acid; RER, respiratory exchange ratio; RIPK, receptor-interacting protein kinase; SR, Sirius red; TNF $\alpha$, tumor necrosis factor alpha.

Word Count: title 107 characters, abstract 275 words, manuscript 6093 (with abstract, methods, figure legends, and references). 


\begin{abstract}
Background \& Aims: In non-alcoholic fatty liver disease (NAFLD), hepatocytes can undergo necroptosis, a regulated form of necrotic cell death mediated by the receptorinteracting protein kinase (RIPK) 1 . We herein assessed the potential of RIPK1 and its downstream effector mixed lineage kinase domain-like protein (MLKL), as therapeutic targets and markers of activity in NAFLD. Methods: C57/BL6J-mice were fed a normal chow diet (NCD) or high fat diet (HFD). The effect of RIPA-56, a highly specific inhibitor of RIPK1, was evaluated in either a prophylactic or a curative treatment of HFD-fed mice, and in primary human steatotic hepatocytes. RIPK1 and MLKL concentrations were measured in the serum of patients with NAFLD. Results: Both prophylactic and curative treatments of HFD-fed mice with RIPA-56, caused a down-regulation of MLKL and a reduction of liver injury, inflammation and fibrosis, characteristic of non-alcoholic steatohepatitis (NASH), as well as of steatosis. This latter effect was reproduced by treating primary human steatotic hepatocytes with RIPA-56 or necrosulfonamide (NSA), a specific inhibitor of human MLKL, and by knocking out (KO) MLKL in fat-loaded AML-12 mouse hepatocytes. MLKL KO in steatotic hepatocytes, caused an activation of the mitochondrial respiration, and an increase in $\beta$-oxidation. Along with MLKL decreased activation, RIPK3-KO mice exhibited increased activities of the liver mitochondrial respiratory chain complexes in experimental NASH. In patients with NAFLD, serum concentrations of RIPK1 and MLKL increased in correlation with the activity. Conclusion: The inhibition of RIPK1 improves NASH features in HFD-fed mice and reverses steatosis by an MLKL-dependent mechanism that involves at least partly an increase in mitochondrial respiration. RIPK1 and MLKL are potential serum markers of activity and promising therapeutic targets in NAFLD.
\end{abstract}




\section{Introduction}

Non-alcoholic fatty liver disease (NAFLD) has been paralleling the worldwide increase in obesity for the past decades. It has become the most common chronic liver disease, and now affects up to one third of the adult population in Western countries [1]. NAFLD encompasses a continuum of entities that span from plain steatosis to non-alcoholic steatohepatitis (NASH), cirrhosis and ultimately end-stage liver disease [2]. Hepatocyte cell death is a critical event in the progression of all chronic inflammatory liver diseases including NAFLD [3]. Until recently, two main forms of cell death were recognized: apoptosis, which occurs in a highly controlled manner, and necrosis that is accidentally triggered. However, during the past few years, it became clear that programmed cell death was not restricted to apoptosis, and comprised other forms of regulated cell death [4]. Necroptosis is one of them, combining the molecular machinery of the extrinsic apoptotic pathways with an execution similar to necrosis [4]. Unlike apoptosis that requires the activation of aspartate-specific proteases known as caspases [5], necroptosis is driven by the activation of the receptor-interacting protein kinase (RIPK) 1 and 3, and the pseudo kinase mixed lineage kinase domain-like (MLKL) [4]. Previous studies have shown that ablation of RIPK3 protected mice from the development of steatohepatitis and fibrosis in a methionine and choline-deficient (MCD) diet mouse model [6, 7]. In addition, it has been demonstrated that necroptosis was activated in the hepatocytes of patients with NASH [6-8]. Overall, compelling studies have shown that RIP kinases were pleiotropic modulators of cell death and participated in the pathogenesis of many chronic diseases [9]. Therefore, there is evidence to suggest that by preventing the formation and/or activation of the necrosome, one could limit cell death and possibly disease progression in NASH.

At present, there are limited options to inhibit the necroptosis pathway. Stable inhibitors that may specifically target RIPK3 or MLKL in vivo, are lacking or induce undesirable effects [10]. The RIPK1 inhibitor necrostatin-1 (Nec-1) has been recognized as a potent inhibitor of necroptosis [11]. However, it is poorly specific, as it also inhibits the indoleamine 2,3-dioxygenase (IDO) pathway. More recently, RIPA-56 was identified as a highly specific and metabolically stable inhibitor of RIPK1 [12].

In the present study, we investigated whether inhibiting RIPK1 would improve NASH features. Specifically, we tested the effect of RIPA-56, in human cell and murine models of steatosis and steatohepatitis. We also explored a hitherto undescribed interaction between the necroptosis pathway and mitochondrial respiration, as a new targetable mechanism of fat 
accumulation. In addition, we analyzed if the mediators of necroptosis might serve as markers of activity in NAFLD. 
Methods (also see Supplementary data for additional Materials \& Methods)

\section{RIPA-56 feeding experiment}

Six-week-old male C57BL/6J mice (Charles River Laboratories, Ecully, France) were fed a high-fat diet (HFD - $45 \mathrm{kcal} \%$ fat) or a normal chow diet (NCD) (Ssniff spezialdiäten GmbH, Soest, Germany) for 16 weeks (the time necessary for an inflammatory and fibrotic response to develop). The effects of a highly-potent and highly-specific RIPK1 kinase inhibitor (referred as to RIPA-56) were evaluated by incorporating it into HFD at $300 \mathrm{mg} / \mathrm{kg}$ dose as initially described [13]. (see Supplementary data for details).

\section{Patients}

Serum samples were obtained from 35 subjects with NAFLD (Supplementary Table 1). The study population was divided in two groups based on the histological score of activity, i.e., the sum of hepatocyte ballooning and lobular inflammation [14]. The first group included subjects with a score $<2$, and the second, subjects with a score $\geq 2$. Human samples were processed and stored by the Biological Resource Center, Bio-ICAN, Institute of Cardiometabolism and Nutrition (IHU-ICAN, ANR-10-IAHU-05), Paris, France (https://www.ican-institute.org/bio-ican-2/).

\section{Isolation and culture of primary human hepatocyte (PHH)}

Ethical approval for the isolation of human hepatocytes was granted by the Persons Protection Committee (CPP Ile de France III) and by the French Ministry of Health (N': COL 2929 and COL 2930). Liver tissue was obtained from subjects undergoing partial hepatectomy for the treatment of colorectal cancer metastases. Cell isolation was performed on Human HepCell platform (IHU-ICAN, Paris, France, http://www.ican-institute.org/category/plateformes), as previously described [15] (See Supplementary data for details).

\section{Real-time quantitative PCR (RT-qPCR)}

Total RNA was extracted using RNeasy columns (Qiagen, Courtaboeuf, France). The mRNA levels of selected genes (for primers, see Supplementary Table 2) were calculated after normalization to Hprt, Hmbs or GAPDH by using the $\triangle \triangle \mathrm{Ct}$ method (See Supplementary data for details). 


\section{Statistical analysis}

Student $t$ test or analysis of variance (ANOVA) were used to compare two groups and three or more groups, respectively. GraphPad Prism software (version 6.0) was used to calculate statistical significance. Statistical tests were used as described in the Figure legends and statistical significance was indicated as follows: ${ }^{*} \mathrm{p}<0.05 ; * * \mathrm{p}<0.01 ; * * * \mathrm{p}<0.001 ; * * * * \mathrm{p}$ $<0.0001$; n.s., not significant. All data are expressed as mean $\pm \mathrm{SEM}$. 


\section{Results}

\section{RIPK1 inhibitor reduces necro-inflammatory and fibrotic NASH features in HFD-fed mice}

RIPA-56 is a highly potent, selective and metabolically stable RIPK1 inhibitor, able to prevent MLKL activation and MLKL-mediated cell death [12], as we herein confirmed using a well-established cell model of TNF $\alpha$-induced cell death, i.e., L929 cells [16] (Supplementary Fig. 1A-C). To explore the therapeutic potential of RIPK1 inhibition in NASH, we tested RIPA-56 in a HFD mouse model. Six-week-old male C57BL/6J mice were fed NCD or HFD for 16 weeks. HFD-fed mice received no additional treatment or RIPA-56, which was administered either from the beginning of HFD feeding or 12 weeks later, to mimic prophylactic and curative treatments, respectively (Fig. 1A). Western blot analyses showed that MLKL and RIPK3, the downstream targets of RIPK1, were overexpressed in the liver of HFD-fed mice compared to NCD-fed mice. In HFD-fed mice RIPA-56 repressed their expression in both treatments, with an almost complete inhibition in the prophylactic setting (Fig. 1B). Likewise, mRNA levels of MLKL and RIPK3 were decreased in both regimens (Supplementary Fig. 2A). In contrast, the expression of RIPK1 was not impacted by RIPA56 treatment (Fig. 1B and Supplementary Fig. 2). The increase of serum alanine aminotransferase (ALT) in HFD-fed mice ( 5-fold increase compared with NCD-fed mice) was abolished by RIPA-56 in both prophylactic and curative settings suggesting decreased tissue damage in these animals (Fig. 1C).

Histological changes of the liver in HFD-fed mice combined steatosis, inflammatory cell infiltrates and fibrosis (Fig. 1D-I). Hepatic F4/80 immunostaining confirmed macrophage infiltration in HFD-fed mice ( $\sim 10$-fold increase compared with NCD-fed mice), which was suppressed by RIPA-56 in both prophylactic and curative treatments (Fig. 1D-E). In keeping with this observation, the mRNA levels of F4/80 and those of Mcp-1, a potent macrophage chemoattractant, were significantly higher in the liver of HFD-fed mice than in NCD-fed mice ( $\sim 3$-fold higher each), and this difference disappeared following both regimens with RIPA-56 (Fig. 1F). The expression of TNF $\alpha$, a major pro-inflammatory cytokine, was increased in the liver of HFD-fed mice as well (3-fold increase), but not after RIPA-56 prophylactic treatment, and markedly reduced after curative treatment (Fig. 1F). Likewise, increments in mRNA levels of other inflammatory markers such as Ccl20, Nlrp3 or $\mathrm{Il}-1 \mathrm{~b}$ observed in the liver of HFD-fed mice, were abrogated or reduced in RIPA-56-treated mice (Supplementary Fig. 
3A). Of note, the levels of intrahepatic and circulating cytokines such as TNF $\alpha$ and MCP-1 that were increased in HFD-fed mice, were lower in RIPA-56-treated mice suggesting that RIPA-56 might reduce inflammation in these animals (Supplementary Fig. 3B and C).

Sirius red staining of liver tissue sections showed that HFD-fed mice developed pericellular fibrosis (10-fold higher than NCD-fed mice), which virtually disappeared following RIPA-56 treatments (Fig. 1G-H). Collagen-1al (Collal) mRNA levels were also increased in the liver of HFD-fed mice compared to NCD-fed mice ( $\sim 6$-fold increase). They were no longer increased after RIPA-56 prophylactic treatment, and reduced after curative treatment (Fig. 1I). These data conclusively demonstrated that RIPK1 contributes to the pathogenesis of NASH, and that a prophylactic treatment with the RIPK1 inhibitor RIPA-56 prevents the histologic features of $\mathrm{NASH}$, whereas a curative treatment largely attenuates their intensity.

\section{RIPK1 inhibitor reverses steatosis and dampens body weight gain in HFD-fed mice}

HFD-fed mice developed obesity. In a prophylactic setting, when RIPA-56 treatment and HFD were started concomitantly, the mice while still obese gained significantly less body weight than untreated HFD-fed mice $(\sim 12 \%$ lower) (Fig. 2A). These mice displayed a decrease in fat and relative fat mass and an increase in lean and relative lean body mass compared to untreated mice under HFD (Supplementary Fig. 4). By contrast, in a curative setting, the delayed start of RIPA-56 after 12 weeks of HFD did not result in differences in body weight gain, fat- or lean-mass compared with untreated HFD-fed mice (Fig. 2A and Supplementary Fig. 4). No significant difference in food intake, spontaneous locomotor activity or respiratory exchange ratio $\left(\mathrm{VCO}_{2}\right.$-to- $\mathrm{VO}_{2}$ ratio) was observed between HFD-fed groups (Fig. 2B-D). Yet, using indirect calorimetry, we noted that energy expenditure was increased in HFD-fed mice, following RIPA-56 prophylactic treatment as compared to those that received RIPA-56 curative treatment or no treatment (Fig. 2E), which could have at least partly contributed to lower body weight gain in these mice. Interestingly, even though HFDfed mice remained obese after both curative and prophylactic RIPA-56 administration, steatosis was largely reduced in both settings (Fig. 2F-H). RIPA-56 caused a marked decrease in hepatic fat content as measured by blinded histological analyses (Fig. 2F-G) and by hepatic triglyceride content ( $\sim 30-40 \%$ reduction) (Fig. 2H). Collectively, these findings indicated that RIPK1 inhibition improved HFD-induced hepatic steatosis.

RIPK1 inhibition promotes fat depletion in primary human steatotic hepatocytes 
To test whether RIPA-56 could directly act on hepatocytes, we first used a model of primary human steatotic hepatocytes. Hepatocytes isolated from the liver of patients with NAFLD were treated after 48 hours of primary culture with RIPA-56 or vehicle (DMSO) for 24 hours. Intracellular lipid content was assessed by Oil Red-O staining and triglyceride assay (Fig. 3AC). RIPA-56-treated steatotic hepatocytes underwent a marked decrease in intracellular lipid droplets (Fig. 3A-B) and triglyceride content (Fig. 3C). To gain insight into the underlying mechanisms, we used primary human hepatocytes, in which steatosis was induced by incubation with a free fatty-acid mixture (oleic acid and palmitic acid, in a molar ratio of 2:1), for 48 hours. Free fatty acid-induced lipid droplets increased by approximately 2- to 3-fold within 48 hours without affecting cell viability (Supplementary Fig. 5A-D). Akin to hepatocytes isolated from steatotic liver, primary human hepatocytes in which steatosis was induced in vitro, underwent a significant decrease in intracellular lipid droplets (Fig. 3D-E) and triglyceride content (Fig. 3F) in response to RIPA-56. This coexisted with an upregulation of $C P T 1 A, A P O B 100$ and $M T T P$ expression, suggesting that increased fatty acid $\beta$ oxidation and/or triglyceride export contributed to the anti-steatotic action of RIPA-56 (Fig. 3G).

\section{MLKL, a downstream target of RIPK1, regulates triglyceride content in hepatocytes}

MLKL is a downstream target of RIPK1, the phosphorylation and activation of which are inhibited by RIPA-56 within hours (Supplementary Fig. 1). Also, the expression of MLKL is down-regulated in the liver of HFD-fed mice after several weeks of RIPA-56 treatment (Fig. 1B and Supplementary Fig.2). Therefore, we hypothesized that MLKL inhibition may be accountable for the fat depletion induced by RIPA-56 in steatotic hepatocytes and tested whether necrosulfonamide, a specific inhibitor of human MLKL, reduces cellular fat in human steatotic hepatocytes. Figure 4 shows that necrosulfonamide significantly decreased intracellular lipid droplets (Fig. 4A-B) and triglyceride content (Fig. 4C), and an upregulated CPT1A expression (Fig. 4D). We also sought to determine if MLKL ablation would reproduce the defatting effect seen in hepatocytes exposed to necrosulfonamide. We knocked out (KO) MLKL using CRISPR-Cas9, in immortalized murine hepatocytes (AML-12 cell line), in which fatty acid metabolism closely resembles that of primary hepatocytes [17]. Efficient KO was confirmed by western blot analysis (Fig. 4E). Following 48-hour incubation with free fatty acids, intracellular triglyceride accumulation was significantly lower in MLKL-KO cells than in controls (Fig. 4F-H). Overall, these findings indicate that destabilizing the necrosome by inhibiting RIPK1 or the final executioner MLKL, or by 
removing MLKL, is sufficient to decrease triglyceride content in hepatocytes. Thus, the RIPK1/MLKL axis appears to be a major pathway of hepatic lipid accumulation in NAFLD.

\section{MLKL regulates mitochondrial biomass and activity}

To gain further insight into the mechanisms whereby the RIPK1/MLKL axis controls fat storage in hepatocytes, we examined mitochondrial activity in MLKL-KO cells. MLKL-KO cells exhibited an apparent increase in viability, as assessed by the MTT assay (Fig. 5A). This effect was independent of proliferation, which was not different between $\mathrm{KO}$ and control cells, as shown by BrdU assay (Fig. 5B) and xCELLigence real-time cell analysis (Fig. 5C). MTT is reduced in metabolically active cells, in part by the action of the mitochondrial dehydrogenase enzyme. We measured the mitochondrial mass and found a moderate but significant increase in KO cells, as compared to controls (Fig. 5D). Likewise, the mRNA levels of Pgcla, a master regulator of mitochondrial biogenesis, were significantly increased in $\mathrm{KO}$ cells (Fig. 5E). Based on these findings, we anticipated that the mitochondrial activity would be increased in $\mathrm{KO}$ cell line. We measured the expression of genes involved in fatty acid $\beta$-oxidation (Cpt1a, Acoxl), and found that they were overexpressed in KO cells, compared to controls (Fig. 5E). We also compared the bioenergetic status of these cells, and performed mitochondrial respiration tests. MLKL-KO cells had a markedly higher basal mitochondrial respiration than control cells (Fig. 5F-G). To distinguish oxygen consumption devoted to ATP synthesis from that due to the natural proton leak across the inner mitochondrial membrane, we added the ATP synthase inhibitor oligomycin, which showed that ATP-linked respiration was increased in KO cells (Fig. 5F-G). The addition of the accelerator ionophore FCCP, which leads to a rapid consumption of oxygen without the generation of ATP, showed that KO cells had a markedly higher maximum respiratory rate than control cells (Fig. 5F-G).

Finally, we assessed whether RIPK3-KO mice with NASH induced by a cholinedeficient, amino acid-defined (CDAA) diet, would also display improved mitochondrial bioenergetics as compared with WT mice (Supplementary Fig. 6A-C). First, we found that RIPK3-KO mice had significantly lower levels of phosphorylated MLKL (p-MLKL) compared with WT mice fed the same diet (Supplementary Fig. 6A). Along with MLKL decreased activation, activities of citrate synthase (CS) and mitochondrial respiratory chain (MRC) complexes in liver mitochondria, particularly the complex II+III, were significantly enhanced in RIPK3-KO mice compared with WT mice (Supplementary Fig. 6B). These differences between WT and KO mice were even more profound with a longer feeding period 
(Supplementary Fig. 6B). Likewise, the mRNA expression levels of Pgcla and Acoxl were both significantly increased in RIPK3-KO mice compared with WT mice at 32 weeks (Supplementary Fig. 6C). Collectively, these data point to a novel function of MLKL activation through the regulation of mitochondrial respiration.

\section{RIPK1 and MLKL are increased in the serum of patients with NASH}

The serum concentrations of proteins mediating necroptosis were previously found to be elevated in patients with tissue injury caused by sepsis $[18,19]$, raising the possibility that this may also occur in patients with NAFLD, as a result of necro-inflammatory activity. We measured the serum concentrations of RIPK1 and MLKL proteins, in 35 patients with NAFLD, and found that their concentration was markedly increased in the serum of patients with active disease (histological activity score $\geq 2, v s .<2$ ) (Supplementary Table 1 and Fig. 6A-B). The serum concentration of both proteins was positively correlated with ALT (Fig. 6C-D). To confirm that mediators of necroptosis might be released in the extracellular milieu during necroptosis, we tested whether L929 cells exposed to TNF $\alpha$, released RIPK1 in their supernatant. We found that RIPK1 was released in the supernatant of L929 cells exposed to $\mathrm{TNF} \alpha$, notably in the presence of Zvad, but remained undetectable, when necroptosis was abrogated by RIPA-56 or Nec-1 treatment of the cells (Fig. 6E). Altogether, these findings suggest that necroptosis contributes to the pathogenesis of NAFLD, and that the release of RIPK1 and MLKL into the systemic circulation, reflects necro-inflammatory activity in human NAFLD. 


\section{Discussion}

The present study provides evidence to indicate that RIPK1 inhibition and subsequent downstream necrosome inactivation improves all histologic features of NASH including liver inflammation and fibrosis. Importantly, we show that RIPK1/MLKL also controls lipid homeostasis and cellular fat deposition. Therefore, the RIPK1/MLKL axis could be a valuable therapeutic target in NAFLD/NASH.

The cell commitment towards apoptosis or necroptosis is largely dependent on the interactions between caspase 8 and RIPK3 [3]. A switch from apoptosis to necroptosis could thus occur whether the necrosome complex is disorganized. Indeed, although RIPK3-deficient mice are protected from MCD diet-induced experimental NASH [6, 7], when RIPK3 is inactivated with the use of a specific kinase inhibitor it may serve as a pro-apoptotic platform leading to the activation of RIPK1-dependent apoptosis in some cellular contexts [10, 20]. Further, while Chenxu et al. showed that invalidating RIPK3 by repeated injections of siRNAs protected mice against HFD-induced hepatosteatosis and metabolic syndrome [21], an excessive sensitivity of hepatocytes and adipocytes to apoptosis has been observed in HFD-fed RIPK3-deficient mice [22, 23]. Noteworthy, although markedly reduced, the expression of RIPK3 was not completely abolished in siRNAs treated mice [21], suggesting that keeping low levels of RIPK3 might be beneficial in HFD experimental model of NASH. Collectively, the therapeutic potential of RIPK3 inhibition in NASH could be cell contextdependent. In contrast, specifically targeting RIPK1, which is upstream of RIPK3 and a key player in cell death controlling both apoptotic and necroptotic signaling pathways should be more effective [24]. Here, we addressed the therapeutic potential of RIPA-56, a specific inhibitor of RIPK1 [12], in HFD-fed mice, both in a prophylactic and curative setting, i.e., after NASH features were already established. RIPA-56 significantly reduced all liver features associated with steatohepatitis, including inflammation and liver fibrosis. While in the prophylactic setting, RIPK1 inhibition slightly reduced HFD-induced weight gain, the overall improvement in liver injury most likely resulted from a direct action rather than being a mere consequence of weight changes.

The complete mechanism underlying necroptosis is still unclear, it was established that the kinase activity of RIPK1 was a prerequisite for the necrosome assembly via phosphorylation, the activation of RIPK3 and its association with MLKL [25]. Further, in this study, we found that RIPA-56 treatment effectively suppressed RIPK3 and MLKL expression while the expression of RIPK1 remained unchanged (Fig. 1B and Supplementary Fig. 2). 
This observation is consistent with other studies showing that chronic inhibition of RIPK1 can lead to reduced expression of RIPK3 and/or MLKL in different inflammatory rodent models [26-29]. Inhibiting the kinase activity of RIPK1 may result in an increase stability of RIPK1. This possibility is even more likely when inhibition is provoked by RIPA-56, which is highly stable in vivo with a half-life of 3.1 hours compared to a half-life < of 5 mins for Necrostatin1 [12]. Finally, as previously suggested [30], it remains theoretically possible that RIPK1 acts as an inhibitor of necroptosis unless its kinase activity is engaged. If this was to be the case, RIPA-56 might stabilize RIPK1 in its inhibitory state, which might lead, in a context of lipid overload, to a downregulation of its downstream targets RIPK3 and MLKL. However, additional investigations are needed to decipher how the inhibition of RIPK1 kinase activity can affect the stability of the necrosome complex.

In addition to key functions in HFD-induced NASH, we herein report that RIPK1 also regulates hepatic steatosis, as shown in the whole liver and in isolated hepatocytes. The mechanism of fat depletion resulting from RIPK1 inhibition, might involve increased mitochondrial activity, which in turn promotes fat mobilization, probably through $\beta$ oxidation. This effect might be mediated through MLKL as we showed that MLKL controls mitochondrial biomass and respiration in cultured AML-12 hepatocytes, and that along with decreased hepatic levels of p-MLKL, RIPK3-KO mice exhibited increased activities of the liver mitochondrial respiratory chain complexes in experimental NASH, as compared with WT mice. Moreover, we found that the absence of MLKL promoted a significant reduction of triglyceride content in fat-loaded AML-12 hepatocytes, and pharmacological inhibition of MLKL by NSA treatment in fat-loaded PHH, reduced the cellular triglyceride content as efficiently as RIPA-56 treatment. Therefore, MLKL appears as a candidate target to restore mitochondrial function in NAFLD.

Interestingly, patients with NASH display hepatic mitochondrial dysfunction, characterized by a decrease in maximal respiration despite an increase in mitochondrial mass [31]. Consequently, the inhibition of the necrosome pathways might also prove beneficial by restoring mitochondrial respiration in patients with NASH. This might result in less steatosis in addition to reduced liver injury and fibrosis as shown here in rodent models. Since an overall reduction of steatosis could result in less lipotoxic lipid species this could also contribute to an anti-fibrotic response [32].

Another intriguing finding is that RIPA-56 prophylactic treatment caused a decrease in weight gain. This finding is in agreement with a recent study showing that MLKL ablation protected mice against obesity [33]. In this study, Xu et al. showed that inhibition of RIPK1, 
RIPK3 or MLKL, by RNAi or pharmacological inhibitors enhanced insulin signaling in vitro and in vivo [33]. These data are in line with our cellular model showing that the ablation of MLKL also increased insulin signaling sensitivity in AML-12 cells (Supplementary Fig. 7). Altogether, this suggests that pharmacological inhibition of the necrosome would not only provide benefits by lowering the intracellular triglycerides stores in steatotic hepatocytes but would also reduce insulin resistance in patients with NAFLD [34].

The clinical relevance of our experimental data is supported by the fact that in a small population of patients with NAFLD, the serum concentrations of RIPK1 and MLKL increased with histological activity and correlated with aminotransferase levels. These findings suggest that a blood-based non-invasive assessment of necroptosis could be useful to identify among patients with NAFLD those who develop active steatohepatitis. However, dedicated studies including a large number of patients are necessary to address the potential interest of measuring markers of necroptosis in serum.

In summary, we have shown that inhibiting RIPK1 and the necrosome pathway in dietary models caused a significant improvement in NASH features in vivo, including steatosis, liver inflammation and fibrosis. We uncovered a novel function of RIPK1/MLKL pathway in the regulation of mitochondrial respiration, which contributes to fat accumulation, notably in hepatocytes. The systemic release of necrosome proteins in patients displaying disease activity supports the clinical importance of this pathway in NASH. Therefore, necroptosis emerges as a major contributor to NAFLD/NASH pathogenesis, and a potential therapeutic target in this disease. 


\section{References}

[1] Younossi Z, Anstee QM, Marietti M, Hardy T, Henry L, Eslam M, et al. Global burden of NAFLD and NASH: trends, predictions, risk factors and prevention. Nat Rev Gastroenterol Hepatol 2018;15:11-20.

[2] Friedman SL, Neuschwander-Tetri BA, Rinella M, Sanyal AJ. Mechanisms of NAFLD development and therapeutic strategies. Nat Med 2018;24:908-922.

[3] Luedde T, Kaplowitz N, Schwabe RF. Cell death and cell death responses in liver disease: mechanisms and clinical relevance. Gastroenterology 2014;147:765-783 e 764.

[4] Vanden Berghe T, Linkermann A, Jouan-Lanhouet S, Walczak H, Vandenabeele P. Regulated necrosis: the expanding network of non-apoptotic cell death pathways. Nat Rev Mol Cell Biol 2014;15:135-147.

[5] McIlwain DR, Berger T, Mak TW. Caspase functions in cell death and disease. Cold Spring Harb Perspect Biol 2013;5:a008656.

[6] Afonso MB, Rodrigues PM, Carvalho T, Caridade M, Borralho P, Cortez-Pinto H, et al. Necroptosis is a key pathogenic event in human and experimental murine models of nonalcoholic steatohepatitis. Clin Sci (Lond) 2015;129:721-739.

[7] Gautheron J, Vucur M, Reisinger F, Cardenas DV, Roderburg C, Koppe C, et al. A positive feedback loop between RIP3 and JNK controls non-alcoholic steatohepatitis. EMBO Mol Med 2014;6:1062-1074.

[8] Gautheron J, Vucur M, Luedde T. Necroptosis in Nonalcoholic Steatohepatitis. Cell Mol Gastroenterol Hepatol 2015;1:264-265.

[9] He S, Wang X. RIP kinases as modulators of inflammation and immunity. Nat Immunol 2018;19:912-922.

[10] Mandal P, Berger SB, Pillay S, Moriwaki K, Huang C, Guo H, et al. RIP3 induces apoptosis independent of pronecrotic kinase activity. Mol Cell 2014;56:481-495.

[11] Takahashi N, Duprez L, Grootjans S, Cauwels A, Nerinckx W, DuHadaway JB, et al. Necrostatin-1 analogues: critical issues on the specificity, activity and in vivo use in experimental disease models. Cell Death Dis 2012;3:e437.

[12] Ren Y, Su Y, Sun L, He S, Meng L, Liao D, et al. Discovery of a Highly Potent, Selective, and Metabolically Stable Inhibitor of Receptor-Interacting Protein 1 (RIP1) for the Treatment of Systemic Inflammatory Response Syndrome. J Med Chem 2017;60:972-986.

[13] Li D, Meng L, Xu T, Su Y, Liu X, Zhang Z, et al. RIPK1-RIPK3-MLKL-dependent necrosis promotes the aging of mouse male reproductive system. Elife 2017;6.

[14] Bedossa P, Consortium FP. Utility and appropriateness of the fatty liver inhibition of progression (FLIP) algorithm and steatosis, activity, and fibrosis (SAF) score in the evaluation of biopsies of nonalcoholic fatty liver disease. Hepatology 2014;60:565-575.

[15] Aoudjehane L, Podevin P, Scatton O, Jaffray P, Dusanter-Fourt I, Feldmann G, et al. Interleukin-4 induces human hepatocyte apoptosis through a Fas-independent pathway. FASEB J 2007;21:1433-1444.

[16] Vercammen D, Beyaert R, Denecker G, Goossens V, Van Loo G, Declercq W, et al. Inhibition of caspases increases the sensitivity of L929 cells to necrosis mediated by tumor necrosis factor. J Exp Med 1998;187:1477-1485.

[17] Nagarajan SR, Paul-Heng M, Krycer JR, Fazakerley DJ, Sharland AF, Hoy AJ. Lipid and glucose metabolism in hepatocyte cell lines and primary mouse hepatocytes: a comprehensive resource for in vitro studies of hepatic metabolism. Am J Physiol Endocrinol Metab 2019;316:E578-E589. 
[18] Sureshbabu A, Patino E, Ma KC, Laursen K, Finkelsztein EJ, Akchurin O, et al. RIPK3 promotes sepsis-induced acute kidney injury via mitochondrial dysfunction. JCI Insight 2018;3.

[19] Wang B, Li J, Gao HM, Xing YH, Lin Z, Li HJ, et al. Necroptosis regulated proteins expression is an early prognostic biomarker in patient with sepsis: a prospective observational study. Oncotarget 2017;8:84066-84073.

[20] Orozco S, Oberst A. RIPK3 in cell death and inflammation: the good, the bad, and the ugly. Immunol Rev 2017;277:102-112.

[21] Chenxu G, Minxuan X, Yuting Q, Tingting G, Jing F, Jinxiao L, et al. Loss of RIP3 initiates annihilation of high-fat diet initialized nonalcoholic hepatosteatosis: A mechanism involving Toll-like receptor 4 and oxidative stress. Free Radic Biol Med 2019;134:23-41.

[22] Gautheron J, Vucur M, Schneider AT, Severi I, Roderburg C, Roy S, et al. The necroptosis-inducing kinase RIPK3 dampens adipose tissue inflammation and glucose intolerance. Nat Commun 2016;7:11869.

[23] Roychowdhury S, McCullough RL, Sanz-Garcia C, Saikia P, Alkhouri N, Matloob A, et al. Receptor interacting protein 3 protects mice from high-fat diet-induced liver injury. Hepatology 2016;64:1518-1533.

[24] Christofferson DE, Li Y, Yuan J. Control of life-or-death decisions by RIP1 kinase. Annu Rev Physiol 2014;76:129-150.

[25] Cho YS, Challa S, Moquin D, Genga R, Ray TD, Guildford M, et al. Phosphorylationdriven assembly of the RIP1-RIP3 complex regulates programmed necrosis and virus-induced inflammation. Cell 2009;137:1112-1123.

[26] Liang YX, Wang NN, Zhang ZY, Juan ZD, Zhang C. Necrostatin-1 Ameliorates Peripheral Nerve Injury-Induced Neuropathic Pain by Inhibiting the RIP1/RIP3 Pathway. Front Cell Neurosci 2019;13:211.

[27] Cui H, Zhu Y, Yang Q, Zhao W, Zhang S, Zhou A, et al. Necrostatin-1 treatment inhibits osteocyte necroptosis and trabecular deterioration in ovariectomized rats. Sci Rep 2016;6:33803.

[28] Liu ZY, Wu B, Guo YS, Zhou YH, Fu ZG, Xu BQ, et al. Necrostatin-1 reduces intestinal inflammation and colitis-associated tumorigenesis in mice. Am $\mathrm{J}$ Cancer Res 2015;5:3174-3185.

[29] Wang JN, Liu MM, Wang F, Wei B, Yang Q, Cai YT, et al. RIPK1 inhibitor Cpd-71 attenuates renal dysfunction in cisplatin-treated mice via attenuating necroptosis, inflammation and oxidative stress. Clin Sci (Lond) 2019.

[30] Linkermann A, Green DR. Necroptosis. N Engl J Med 2014;370:455-465.

[31] Koliaki C, Szendroedi J, Kaul K, Jelenik T, Nowotny P, Jankowiak F, et al. Adaptation of hepatic mitochondrial function in humans with non-alcoholic fatty liver is lost in steatohepatitis. Cell Metab 2015;21:739-746.

[32] Pelusi S, Valenti L. Hepatic fat as clinical outcome and therapeutic target for nonalcoholic fatty liver disease. Liver Int 2019;39:250-256.

[33] Xu H, Du X, Liu G, Huang S, Du W, Zou S, et al. The pseudokinase MLKL regulates hepatic insulin sensitivity independently of inflammation. Mol Metab 2019.

[34] Marchesini G, Brizi M, Morselli-Labate AM, Bianchi G, Bugianesi E, McCullough AJ, et al. Association of nonalcoholic fatty liver disease with insulin resistance. Am J Med 1999;107:450-455. 


\section{Figure legends}

Figure 1. RIPA-56 treatment improves liver inflammation and fibrosis in HFD-fed mice. Six-week-old male C57BL/6J mice ( $n=5$ per group) were fed either with normal control diet (NCD) or high-fat diet (HFD) supplemented or not with RIPA-56, according to prophylactic ("Pro") or curative ("Cur") designs for 16 weeks to induce a sufficient inflammatory and fibrotic response to HFD. Differences between mice were determined by analysis of variance (ANOVA) with Bonferroni's multiple comparison. (A) Schematic representation of the experimental design; (B) Immunoblot analyses of whole liver protein extracts from mice fed NCD (1 to 3), HFD (4 to 6), HFD with RIPA-56 "Pro" (7 to 9), or HFD with RIPA-56 "Cur" (10 to 12) using antibodies against RIPK1, MLKL, RIPK3 and GAPDH as loading control; (C) Serum analysis of ALT; (D) Immuno-histochemical analysis of F4/80 on representative liver tissue sections from mice of each group; (E) Quantitative analysis of $\mathrm{F} 4 / 80^{+}$foci using FIJI software, 8-10 pictures per mouse were quantified, scale bars indicate $50 \mu \mathrm{m}$; (F) F4/80, Mcp-1 and Tnfa mRNA levels were assessed by RT-qPCR. (G) Representative Sirius Red staining of liver tissue section from mice of each group, scale bars indicate $50 \mu \mathrm{m} ;(\mathbf{H})$ Quantification of light polarized Sirius Red pictures, 10 pictures per mouse were analyzed; (I) Colla1 mRNA levels were assessed by RT-qPCR. All data are expressed as mean \pm SEM; *p $<0.05 ; * * \mathrm{p}<0.01 ; * * * \mathrm{p}<0.001$

Figure 2. RIPA-56 treatment improves HFD-induced obesity and steatosis in mice. Sixweek-old male C57BL/6J mice ( $n=5$ per group) were fed either NCD or HFD supplemented or not with RIPA-56 for 16 weeks, as depicted in Fig. 1A, to analyze the metabolic status of the mice. (A-E) Differences between groups were determined by two-way ANOVA with Tukey's multiple comparison. (G-H) Differences between groups were determined by oneway ANOVA with Bonferroni's multiple comparison. (A) Body weight gain in mice of the 4 groups. No significant change in (B) food intake, $(\mathbf{C})$ spontaneous locomotor activity, or (D) respiratory exchange ratio (RER) between HFD-fed mice. \# indicates that changes in NCDfed mice are significantly higher than HFD-fed mouse groups; (E) Whole energy expenditure was evaluated during daylight and night in mice of the 4 groups. $\$$ indicates that energy expenditure in NCD-fed mice is significantly increased compared with HFD and curative mouse groups but identical compared with prophylactically treated mice; $(\mathbf{F})$ Representative H\&E staining of liver tissue sections from mice of the 4 groups. Scale bars, $50 \mu \mathrm{m} ;(\mathbf{G})$ 
Steatosis score of liver tissue sections was evaluated blinded; (H) Intrahepatic triglyceride contents were measured in two different liver samples per mouse. All data are expressed as mean \pm SEM; $* \mathrm{p}<0.05 ; * * \mathrm{p}<0.01 ; * * * \mathrm{p}<0.001 ; * * * * \mathrm{p}<0.0001 ;$ n.s., not significant.

Figure 3. RIPA-56 treatment induces fat depletion in primary human hepatocytes. Primary human hepatocytes were isolated from steatotic human livers of patients with NAFLD (A-C) $(n=5)$ or from non-steatotic human livers $(n=5)$ and incubated with free fatty acids to induce steatosis or without (W/O) (D-G), and treated with RIPA-56 (20 $\mu \mathrm{M})$ or DMSO (vehicle) for 24h. Differences between two groups were determined using Student's $t$ test. (A, D) Representative Oil Red-O and DAPI images, scale bars indicate $100 \mu \mathrm{m} ;(\mathbf{B}, \mathbf{E})$ Quantification of Oil Red-O staining normalized for the number of DAPI-stained nuclei; $(\mathbf{C}$, F) Quantification of intracellular triglycerides normalized to protein content; (G) mRNA levels of $C P T 1 A, M T T P$ and $A P O B 100$ were assessed by RT-qPCR, and shown related to DMSO-treated hepatocytes. All results are shown as mean \pm SEM; n.s., not significant; ${ }^{*} \mathrm{p}<$ $0.05 ; * * p<0.01$. These findings are representative of five independent cell preparations.

Figure 4. MLKL controls intrahepatic triglyceride content in hepatocytes. (A-D) Primary human hepatocytes were isolated from non-steatotic human livers $(n=5)$ and incubated with free fatty acids to induce steatosis, or without (W/O), and treated with necrosulfonamide (NSA) $(20 \mu \mathrm{M})$ or DMSO (vehicle) for $24 \mathrm{~h}$. Differences between groups were determined by one-way ANOVA with Bonferroni's multiple comparison; (A) Representative Oil Red-O and DAPI images, scale bars indicate $100 \mu \mathrm{m}$; (B) Quantification of Oil Red-O staining normalized for the number of DAPI-stained nuclei indicates; (C) Quantification of intracellular triglycerides normalized to protein content. \# indicates that changes are significantly higher than hepatocytes W/O FFA (D) mRNA levels of CPT1A, MTTP and $A P O B 100$ were assessed by RT-qPCR, and shown related to DMSO-treated hepatocytes; (EH) AML-12 cells were knocked-out (KO) for MLKL and compared with control (CTL) cells, which underwent a similar CRISPR-Cas9 selection as KO cells. Differences between CTL and KO cells were determined using Student's $t$-test; (E) Whole-cell lysates were extracted from CTL and MLKL-KO AML-12 cells and analyzed by Western blot using antibodies against MLKL, Tubulin and GAPDH; (F) Representative Oil Red-O and DAPI images; (G) Oil Red-O staining and $(\mathbf{H})$ triglycerides quantification in fat-loaded cell lines. All data are expressed as mean $\pm \mathrm{SEM}$; n.s., not significant; ${ }^{*} \mathrm{p}<0.05 ;{ }^{*} \mathrm{p}<0.01 ; * * \mathrm{p}<0.001$. These experiments are representative of five independent cell preparations. 
Figure 5. MLKL regulates mitochondrial respiration in murine hepatocytes. (A) Cell viability was analyzed using the MTT assay; Cell proliferation was analyzed using (B) BrdU incorporation and (C) real-time cell analysis (xCELLigence Cim-Plate 96). Each cell line was seeded in six replicate wells; (D) The mitochondrial mass was evaluated using a MitoTracker Red-Probe in triplicate; (E) mRNA levels of Pgcla, Cptla and Acoxl were assessed by RTqPCR in triplicate; (F) The respiratory flux profiles of cells were determined using a Seahorse Extracellular Flux Analyzer with twelve consecutive measurements of oxygen consumption rate (OCR). Each cell line was seeded in eight replicate wells. Bioenergetic parameters including basal (Blue), ATP-linked (Pink), proton leak (Purple), maximal respiration capacity (Green) and non-mitochondrial respiration (Grey) are outlined with corresponding mitochondrial effectors for AML-12 ${ }^{\mathrm{CTL}}$ cells; (G) Basal mitochondrial OCR, ATP-linked OCR, maximum OCR are represented as fold activation. All results are shown as mean \pm SEM; n.s.: not significant; ${ }^{*} \mathrm{p}<0.05 ;{ }^{* *} \mathrm{p}<0.01 ; * * * \mathrm{p}<0.001 ; * * * * \mathrm{p}<0.0001$. Differences between CTL and KO cells were determined using Student's $t$-test. All experiments are representative of three independent cell preparations.

Figure 6. RIPK1 and MLKL are released extracellularly during necroptosis. (A) ELISA analysis of RIPK1 and MLKL protein levels in the serum of subjects with NAFLD and a histological activity score $<2(n=8)$ or $\geq 2(n=27) ;{ }^{*} \mathrm{p}<0.01 ;(\mathbf{B})$ Correlation plot analysis between RIPK1 or MLKL and ALT levels in all subjects, $r^{2}$ values were calculated with Pearson's correlations; (E) ELISA analysis of RIPK1 in the supernatant cleared from debris of L929 cells treated with the pan-caspase inhibitor Zvad $(20 \mu \mathrm{M})$, RIPA-56 $(20 \mu \mathrm{M})$, necrostatin-1 (Nec-1, $20 \mu \mathrm{M})$ or vehicle (DMSO) for $2 \mathrm{~h}$, prior to incubation with or without $\mathrm{TNF} \alpha(25 \mathrm{ng} / \mathrm{mL})$ for $6 \mathrm{~h}$. Results are shown as mean $\pm \mathrm{SEM}$ of six replicates. 


\section{Financial support}

Jérémie Gautheron is funded by the Fondation pour la Recherche Médicale (FRM ARF20170938613), the Institute of Cardiometabolism and Nutrition (ICAN PAP17NECJG), and the Mairie de Paris (Emergences - R18139DD). Amine Majdi is supported by the Ministère de l'Enseignement Supérieur et de la Recherche (MESR). Cecília M. P. Rodrigues is funded by the Fundação para a Ciência e a Tecnologia (PTDC/MEDFAR/29097/2017), and the EU H2020 Marie Sklodowska-Curie 722619 grant.

\section{Acknowledgements}

The authors thank L. Dinard, Q. Pointout, T. Coulais and A. Guyomard from the animal facilities platform (PHEA Saint-Antoine) for their technical support, A. Munier from the cytometry platform for her help in FACS analysis, R. Morichon from the image platform for his help in microscopy analysis, and B. Solhonne and F. Merabtene for their contribution in liver tissue histomorphological analysis. The authors also acknowledge A. Lacombe from the functional and physiological platform (PreclinICAN) for her help in indirect calorimetry. We are also thankful to R. Castro and P. Rodrigues (iMed.ULisboa) for their help with RIPK3 ${ }^{-/-}$ experiments.

\section{Authors contributions}

J.G. conceived and designed the study; A.M., L.A., T.M., and J.G. performed, analyzed and interpreted most of the experiments; F.B. and T.L. provided technical assistance; L.A., F.C. and O.S. designed and performed the primary human hepatocyte experiments; V.R. provided human patients' serum samples and clinical data; T.I. analyzed MRC complex activity; M.B.A. performed RIPK3 KO animal experiments; V.R., F.C., M.L., F.F., M.M., A.C., L.F., T.A-S., J-L.D., G.C., B.F., O.S., C.P-B., C.M.P.R. and C.H. provided important intellectual input; J.G., V.R. and C.H. wrote the manuscript; G.C., B.F. and C.M.P.R. critically revised the manuscript; J.G. obtained funding for the study.

\section{Supporting information}

Supplementary data include Supplementary Methods \& Materials, seven Supplementary Figures, and two Supplementary Tables. 